\title{
Creación de empresas y recuperación económica: El caso de Rio Grande do Sul
}

\author{
Mario Duarte Canever y André Carraro
}

RESUMEN

PALABRAS CLAVE

CLASIFICACIÓN JEL

AUTORES
En este artículo se analiza la dinámica de la creación de nuevas empresas en Rio Grande do Sul mediante la tasa de creación de empresas relativa al conjunto de aquellas ya existentes y la tasa relativa a la fuerza de trabajo. El análisis revela que las tasas de creación de empresas no son homogéneas a nivel regional ni sectorial. Asimismo, se establece que existe una relación entre la dinamicidad económica regional y la tasa de creación de empresas. Esa relación depende del nivel de renovación de la base empresarial y, fundamentalmente, del impulso emprendedor de los trabajadores locales, que influye en la convergencia de la tasa de creación de empresas entre las regiones. Los resultados muestran también que un aumento en la tasa de creación de empresas conduce a un incremento del desempeño regional después de cierto período.

Capacidad empresarial, desarrollo de empresas, condiciones económicas, desarrollo económico, desarrollo regional, datos estadísticos, Brasil

L26, R11, R12

Mario Duarte Canever es profesor del Departamento de Ciencias Sociales Agrarias de la Universidad Federal de Pelotas (UfP). canever@ufpel.edu.br

André Carraro es profesor del Departamento de Economía de la Universidad Federal de Pelotas (UfP). andre.carraro@gmail.com 


\section{I}

\section{Introducción}

Durante más de 20 años, la tasa de creación de nuevas empresas ha sido considerada un factor importante para la formulación de políticas de desarrollo regional. El nacimiento de un nuevo negocio es una valiosa expresión de la actividad emprendedora, que constituye un elemento fundamental para el crecimiento económico (Johnson, 2004). Las variaciones espaciales en la tasa de creación de empresas dentro de un país y entre países plantean reiterados desafíos a los responsables de la formulación de políticas. Los estudios del Global Entrepreneurship Monitor (GEM) revelan las variaciones entre países de manera constante. Por ejemplo, de acuerdo con el estudio del GEM de 2002, que abarca a 37 países, la actividad emprendedora total — definida como el porcentaje de la fuerza de trabajo involucrada en la apertura o en los primeros pasos de una nueva empresa- varió del 18,7\% en Tailandia a apenas un 1,8\% en el Japón (Reynolds, Bygrave y Autio, 2004, pág. 4). En ese mismo estudio se aprecia una gran variación entre las regiones del mundo, con las mayores tasas de creación de empresas registradas en América Latina y en los países en desarrollo de Asia.

La variación espacial también es considerable dentro de cada país. Por ejemplo, Reynolds, Storey y Westhead (1994) encontraron que la relación entre la mayor y la menor tasa de creación de empresas entre las regiones de los Estados Unidos de América era de 4,1. Al calcular la tasa de creación de nuevas empresas de 1994 a 2001 en las diversas regiones del Reino Unido de Gran Bretaña e Irlanda del Norte, Johnson (2004) registró la mayor en la región de Londres y la menor en Irlanda del Norte. Sobre la base de datos municipales del Reino Unido de Gran Bretaña e Irlanda del Norte, Ashcroft, Plotnikova y Ritchie (2007) concluyeron que la tasa de creación de nuevas empresas varía con el tiempo y en el espacio. Según estos autores, el carácter cíclico de la tasa de creación de nuevas empresas refleja las variaciones macroeconómicas del país. La tasa de creación también tiende a variar entre los municipios con el paso de los años y se sugiere que las variaciones son procíclicas. En otras palabras, cuando la economía del país mejora en general, el desempeño relativo de los municipios con una alta tasa de creación de nuevas empresas tiende a ser mejor que el del resto del país.

En consecuencia, no sorprende la importancia de los estudios en que se demuestran empíricamente las variaciones en las tasas de creación de empresas y su repercusión en el desempeño económico regional. Por otra parte, en muchos estudios también se evidencia la relación entre la creación de empresas y la generación de empleos (Hart y Oulton, 2001), la innovación (Audretsch, 1999), el crecimiento económico (Schmitz, 1989), y la reducción del desempleo (Thurik, 1999). Los responsables de la formulación de políticas públicas interesados en mejorar el desempeño económico de una región deberían buscar maneras eficaces de estimular las tasas de creación de nuevas empresas.

Si bien a nivel internacional hay relativamente muchos estudios en que se relaciona la creación de empresas con el aspecto espacial y el desarrollo regional (Reynolds, Storey y Westhead, 1994; Johnson, 2004; Van Stel, Carree y Thurik, 2005; Ashcroft, Plotnikova y Ritchie, 2007), todavía es necesario profundizar el análisis de esta temática en el caso del Brasil. Los estudios de Campos e Iootty (2005); Barros y Pereira (2008), y Canever y otros (2010) constituyen algunas excepciones recientes. En el primero se analizan los factores determinantes de la entrada y la salida de empresas del Brasil sobre la base de datos del Instituto Brasileño de Geografía y Estadística (IBGE) y, entre otros resultados, los autores comprobaron empíricamente la relación entre crecimiento sectorial y entrada neta de empresas. Barros y Pereira (2008) analizaron la influencia de la actividad emprendedora en el producto interno bruto (PIB) y el desempleo en los municipios de Minas Gerais y encontraron que una mayor tasa de espíritu empresarial conduce a una reducción del desempleo, pero no necesariamente a un mejor desempeño económico medido por el crecimiento del PIB local. Por el contrario, Canever y otros (2010) estudiaron los aspectos que preceden a la tasa de creación de empresas en Rio Grande do Sul (RS) y las consecuencias que esta tiene en el desempeño municipal, y hallaron relaciones positivas entre la actividad emprendedora y el nivel del PIB per cápita en los años subsiguientes. Una característica común a esos tres trabajos es la medida de la actividad emprendedora utilizada. Campos e Iootty (2005) emplean la diferencia en el conjunto absoluto de empresas en años consecutivos como medida del espíritu empresarial. Barros y Pereira (2008) miden la tasa de espíritu empresarial mediante la proporción de trabajadores por cuenta propia, mientras 
que Canever y otros (2010) utilizan la diferencia en el conjunto de empresas per cápita con respecto a la población adulta de un municipio en años consecutivos, como valor representativo de la actividad emprendedora. $\mathrm{Si}$ bien dichas medidas pueden estar relacionadas con la creación de empresas, constituyen indicadores de la proporción de empresarios en el total de la población y del crecimiento de la estructura empresarial, pero no de la creación de nuevas empresas.

En el presente estudio se reformula el indicador de la actividad emprendedora, estableciendo una definición conceptual y operacional de la tasa de creación de nuevas empresas a partir de los datos de la Relación Anual de Informaciones Sociales (RAIS), divulgados por el Ministerio del Trabajo y Empleo con respecto a los municipios del estado de Rio Grande do Sul.

Vistas la importancia de la creación de nuevas empresas para el desarrollo regional y la escasez de estudios en que se examina esta relación en el contexto brasileño, en este trabajo se plantearon los siguientes objetivos: i) determinar si las tasas de creación de empresas en Rio Grande do Sul son homogéneas; ii) determinar si las regiones consideradas menos dinámicas presentan tasas de creación de empresas diferentes de la del estado en general, y iii) demostrar la manera en que la tasa de creación de empresas influye en el desarrollo de las regiones.

El estado de Rio Grande do Sul se divide en dos regiones distintas en términos de desarrollo (véase el gráfico 1). La parte norte, que abarca cerca del $80 \%$ de los municipios y el 50\% del territorio, es considerada más desarrollada y más dinámica. El producto per cápita medio en 2008 era de aproximadamente 20.000 reales. Por el contrario, la parte sur, localizada en la frontera con el Uruguay, es menos dinámica y su producto interno bruto medio en 2008 fue de 15.000 reales por habitante. Esos datos describen una dinámica dual de distribución del ingreso en el estado. Hasta

GRÁFICO 1

\section{Regiones de Rio Grande do Sul}

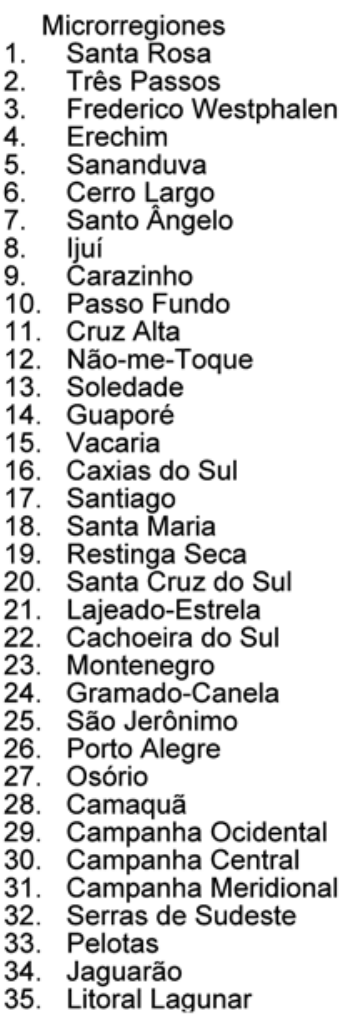
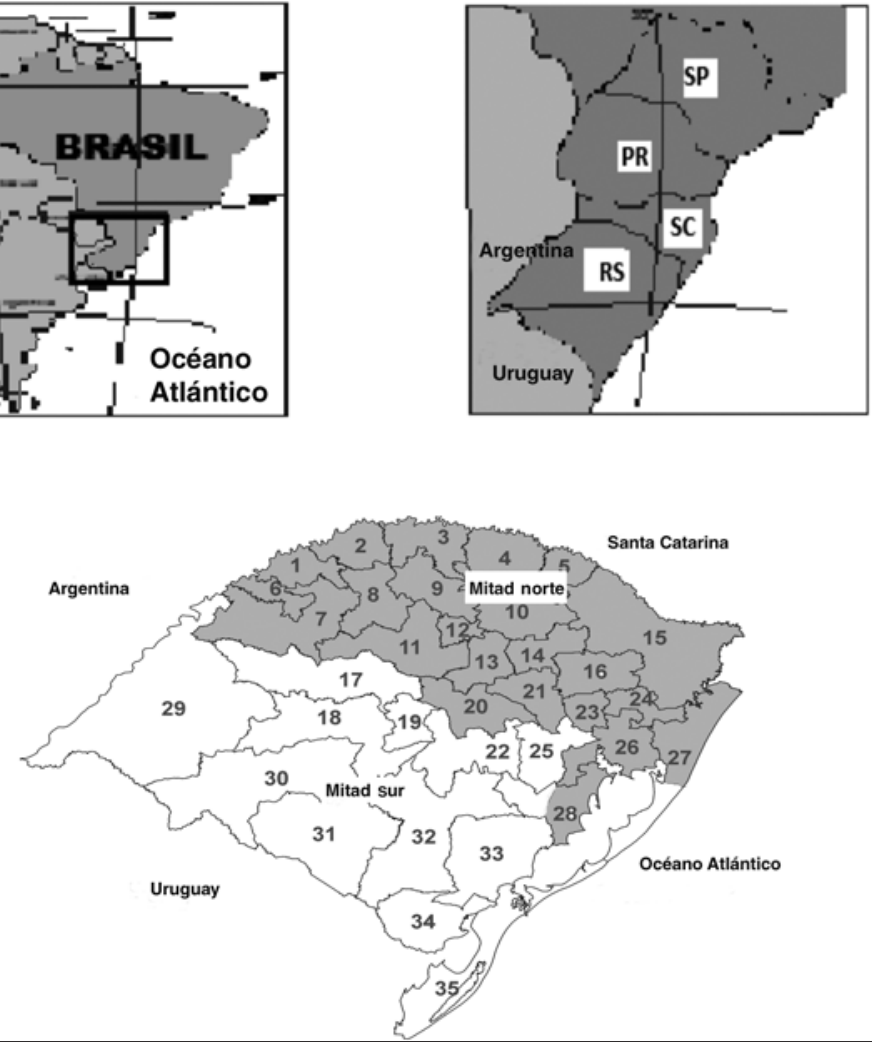

Fuente: elaboración propia sobre la base de http://pt.wikipedia.org/wiki/Rio Grande do Sul. 
el momento se han planteado muchas razones para esa disparidad, pero en ningún estudio se analizaron la tasa de creación de nuevas empresas y el espíritu empresarial como posibles causas. En este marco, Rio Grande do Sul parece ser un caso especial para ilustrar la relación entre la creación de empresas y el crecimiento económico, temática poco explorada en el contexto de los países en desarrollo.
El presente artículo se divide en cinco secciones, incluida esta Introducción. A continuación (sección II) se examina la importancia del espíritu empresarial y de la creación de nuevas empresas para el desarrollo local y en la sección III se presentan los datos y los procedimientos metodológicos utilizados. Mientras que en la sección IV se describen y analizan los resultados, en la $\mathrm{V}$ se formulan las conclusiones.

\section{II \\ Desarrollo, espíritu empresarial y tasa de creación de nuevas empresas}

En la actualidad, existe un gran interés en las interrelaciones entre el espíritu empresarial y el desarrollo económico, y entre dicho espíritu y el desarrollo socialhumano, tanto a nivel académico como entre los gestores de las diversas instancias empresariales de la iniciativa privada y los responsables de la formulación de políticas públicas. Sin embargo, debido a limitaciones de carácter conceptual y metodológico, todavía son escasos los estudios en que se investigan las relaciones entre esas variables (véanse algunas excepciones en Wennekers y Thurik, 1999; Van Stel, Carree y Thurik, 2005). El espíritu empresarial y el desarrollo son fenómenos complejos, difíciles de medir y sus conceptos pueden asumir significados diversos en función de las corrientes teóricas que los sustentan. Esto determina a su vez que la construcción de teorías y pruebas sea también una tarea compleja y difícil ${ }^{1}$.

La relación entre el espíritu empresarial y el desarrollo se descubrió hace bastante tiempo, cuando el economista austriaco Joseph A. Schumpeter (1883-1950) formuló la teoría de los flujos circulares y explicó los factores condicionantes del proceso de desarrollo económico. El desarrollo, en el sentido propuesto por el autor, deriva de la creación innovadora fomentada por los emprendedores.

Después del enfoque pionero de Schumpeter (véase Schumpeter, 1982), los modelos de crecimiento económico evolucionaron a partir de la función de producción neoclásica, en la que el factor de producción era la variable clave para descifrar el enigma del problema

\footnotetext{
1 Véase un análisis del debate sobre el concepto de espíritu empresarial en Herbert y Link (1989); Thorton y Flynne (2003); y OCDE (1998).
}

relacionado con el crecimiento económico. En esta línea se destaca el trabajo precursor de Solow (1956), que propuso un modelo de crecimiento económico basado en los factores de producción capital y trabajo. A pesar de los avances propuestos por Solow (1956), el enfoque crítico de Romer (1986) dirigió la atención de los investigadores del área hacia lo que él consideraba una omisión en el modelo de Solow: el conocimiento. A partir de la contribución de Romer (1986), se realizó una vasta gama de estudios en que se destacaba el papel de la inversión en capital humano, educación e investigación y desarrollo (I+D), en el marco de la llamada teoría del crecimiento endógeno ${ }^{2}$.

A pesar de la evolución teórica y empírica de los nuevos modelos de crecimiento endógeno, más recientemente ha surgido un nuevo conjunto de trabajos, entre ellos los de Garther y Carter (2003) y Audretsch (2007), en los que se sugiere una nueva variable omitida en el modelo pionero de Solow: el propio empresario schumpeteriano. Para esos autores, el espíritu empresarial, entendido como la capacidad de los agentes económicos de generar nuevas empresas, debería incluirse en los modelos de crecimiento económico haciendo explícita una nueva forma de capital: el capital empresarial.

A diferencia de la noción típica del espíritu empresarial como una acción, proceso o actividad profesional, en esta línea de investigación se lo considera como una reserva de capital (Hofstede y otros, 2002). La propuesta no consiste en negar los avances del modelo de Solow,

\footnotetext{
2 Sobre modelos de crecimiento endógeno, véanse Romer (1990); Grossman y Helpman (1991); Aghion y Howitt (1998).
} 
sino en perfeccionarlo mediante la distinción de la variable capital de su modelo en capital físico, capital humano y capital empresarial. Al mismo tiempo, se abre la posibilidad de conciliar dos importantes corrientes de investigación del crecimiento económico, al afirmarse que el capital empresarial permite la aproximación entre el romántico e ingenuo empresario del modelo schumpeteriano y el científico y rígido modelo de (I+D) de Romer (1990) y Grossman y Helpman (1991).

La función de producción compuesta por sus principales factores determinantes puede ilustrarse mediante una típica función de Cobb-Douglas:

$$
Y_{i}=\alpha K_{i}^{\beta} L_{i}^{\delta} R_{i}^{\phi} E_{i}^{\gamma} e_{i}^{\varepsilon}
$$

donde $K$ representa el capital físico, $L$ la fuerza de trabajo, $R$ el capital conocimiento, $E$ el capital empresarial e $i$ representa diferentes regiones.

Aquí, la variable capital físico caracteriza a la capacidad de inversión de una región en la creación de una reserva de capital físico. La fuerza de trabajo $(L)$ corresponde a la fuerza de trabajo necesaria para la función de producción y la variable $R$ representa el conjunto de ideas resultantes del proceso de investigación, que están disponibles para cualquier interesado. Por último, la variable capital empresarial $(E)$ capta el efecto del agente empresario que es capaz de percibir las oportunidades que derivan de un cambio en el ámbito de la demanda o de la oferta. Los exponentes representan sus respectivas productividades marginales que, conforme con la hipótesis de este trabajo, son decrecientes.

Al poner de relieve el capital empresarial se cubre una carencia habitual en este tipo de estudios, pues se explicita el papel del emprendimiento en el proceso de crecimiento económico. Es decir que, $c e-$ teris paribus, la existencia de agentes más dispuestos a correr el riesgo de nuevos negocios permite una mejor utilización del capital físico, la mano de obra y el conocimiento existentes, lo que se traduce en una asignación de recursos más eficiente y promueve el crecimiento económico.

En consecuencia, la apertura de nuevas empresas (capital empresarial) pasa a relacionarse formalmente con el crecimiento económico por, al menos, tres vías distintas (Audretsch, 2007). La primera se refiere al papel del capital empresarial como mecanismo de difusión del conocimiento generado por los modelos de (I+D). Romer (1990), Lucas (1988), y Grossman y Helpman (1991) subrayan la importancia de los efectos secundarios (spillovers) del conocimiento como variable clave en los modelos de crecimiento endógeno. Sin embargo, poco se dice sobre el mecanismo mediante el cual el conocimiento se difunde en la sociedad. Si es verdad que un sistema de innovación está compuesto por la (I+D), también parece cierto que la difusión del conocimiento generado por la inversión en investigación necesita del capital empresarial para constituir el vínculo entre la invención y la innovación. En otras palabras, si en el modelo de Schumpeter de 1912 (Schumpeter, 1982) el empresario innovador es el responsable de la ruptura del flujo circular del ingreso, en el modelo de crecimiento endógeno la responsabilidad de la difusión de la innovación recae en el capital empresarial ${ }^{3}$.

La segunda vía que relaciona el capital empresarial con el crecimiento económico corresponde al efecto competencia. $\mathrm{Al}$ crearse un número mayor de empresas se incrementa el capital empresarial de una región y, al mismo tiempo, aumenta la competencia. Tanto en los modelos microeconómicos (Varian, 1992), como en el modelo de Porter (1991), el conocimiento tiene mayor difusión en las estructuras de mercado competitivas que en los monopolios. Esta teoría se respalda mediante evidencia empírica en los trabajos de Feldmann y Audretsch (1999) y Glaeser y otros (1992), que encontraron una relación positiva entre el crecimiento económico y el acrecentamiento de la competencia, medida por el número de empresas en una ciudad.

Por último, la tercera vía se basa en el efecto diversificación. La presencia de un mayor capital empresarial no solo afecta al crecimiento económico debido al aumento del número de empresas, sino también a causa de la mayor diversificación de empresas en una región. Según el análisis pionero de la economía de las ciudades, de Jacobs (1969), la complementariedad del conocimiento y la información que poseen las diferentes empresas dentro de una región geográfica produce una externalidad positiva que incrementa el rendimiento de las inversiones, la actividad innovadora y el crecimiento económico.

En el caso del Brasil, los estudios en que se relacionan el desarrollo y el espíritu empresarial se concentran en análisis descriptivos de las aglomeraciones productivas o polos tecnológicos existentes en las distintas regiones ${ }^{4}$.

\footnotetext{
${ }^{3}$ De acuerdo con este enfoque, una importante investigación en el área de la biotecnología, con gran potencial de mercado, será solo un informe de investigación hasta que aparezca una empresa dispuesta a difundir el nuevo conocimiento.

4 Véanse, por ejemplo, los estudios encabezados por Haddad (1999); Castilhos (2002); Lastres, Cassiolato y Maciel (2003); Cassiolato y Szapiro (2000); Cassiolato, Lastres y Szapiro (2000), entre otros.
} 
Si bien en dichos estudios se traza el perfil económico y social de diversas regiones donde existen aglomeraciones productivas industriales específicas con potencial de desarrollo local, no se analiza el capital empresarial per se, sino las acciones innovadoras necesarias para que la región se desarrolle. Con respecto al estado de Rio Grande do Sul, el debate sobre la dinámica espacial de la distribución del ingreso permite un análisis más detallado de la distribución de la tasa de creación de empresas (capital empresarial) y su relación con el crecimiento económico 5 .

5 Véase Porsse, Rosa y Porto (2008).

\section{III}

\section{Datos y procedimientos metodológicos}

\section{Datos}

Para estimar las tasas de creación de empresas, en este trabajo se utiliza el número de unidades locales (empresas) según las secciones de clasificación de actividades propuestas en la Relación Anual de Informaciones Sociales (RAIS), divulgada por el Ministerio del Trabajo y Empleo por ciudad (municipio) y microrregiones del estado de Rio Grande do Sul. Los datos abarcan los 496 municipios del estado y corresponden al período de 1995 a 2008. A fin de poder utilizar la base de datos para el cálculo de la tasa de creación de nuevas empresas, se separaron en primer lugar las empresas nuevas (matrices) de las ya existentes (filiales). Se considera que el nacimiento (apertura) de una empresa corresponde a su presencia por primera vez en la base de datos de la RAIS. Así, una empresa que no figuraba en la RAIs en $t-1$ y pasó a figurar en $t$ se considera como una empresa que nació en $t$, ya que fue el primer año en que apareció en la base de datos.

Solo se tuvieron en cuenta las empresas que constaban en la base de datos en años consecutivos o, en otras palabras, no se consideraron los establecimientos que aparecieron en la base de datos en un determinado año, desaparecieron al año siguiente y volvieron a figurar en el año subsiguiente. También se filtraron las empresas que nacieron en un determinado año, desaparecieron por un tiempo mayor $(t+2$, por ejemplo) y reaparecieron en períodos subsiguientes. En virtud de este procedimiento, no se estimaron las tasas relativas al último año de la serie (2008), ante la imposibilidad de utilizar el filtro descrito previamente. Este procedimiento también fue adoptado por Souza y otros (2007) a objeto de limpiar la base de datos para los cálculos de las tasas de creación de empresas que, de lo contrario, resultarían sesgadas. De hecho, se cree que aunque no proporcionen datos, estas empresas todavía permanecen activas. Si bien la población de empresas se redujo cerca de un $1 \%$ al año debido a las mencionadas exclusiones, esto no compromete la validez de los resultados, pues la muestra comprende más de 100.000 empresas cada año.

Para facilitar el análisis se agregaron los datos de los 21 sectores económicos presentados por la RAIS en tres sectores económicos tradicionales, a saber:

i. Agricultura - comprende las empresas en los sectores de agricultura, ganadería, producción forestal, pesca y acuicultura.

ii. Industria - comprende las empresas de la industria extractiva, la industria de transformación y la construcción civil.

iii. Comercio y servicios - comprende las empresas de electricidad y gas; agua, alcantarillado, actividades de gestión de residuos y descontaminación; comercio, reparación de vehículos automotores y motocicletas; transporte, almacenamiento y correo; alojamiento y alimentación; información y comunicación; actividades financieras, de seguros y servicios relacionados; actividades inmobiliarias; actividades profesionales, científicas y técnicas; actividades administrativas y servicios complementarios; administración pública, defensa y seguridad social; educación; salud humana y servicios sociales; artes, cultura, deporte y recreación; otras actividades de servicios; servicios domésticos; organismos internacionales y otras instituciones extraterritoriales.

\section{Procedimientos metodológicos}

La tasa de creación de empresas puede medirse de diversas formas. Considerado el sector de la economía $i$ en la región $r$, la tasa de creación de empresas en dicho sector puede definirse de la siguiente manera: 


$$
\text { Fir }=\frac{\text { NEtir }- \text { NEtir }-1}{\text { NEtir }-1}
$$

donde: $\mathrm{NE}=$ número de empresas del sector $i$ en la región $r . t=$ año.

En la tasa de creación de empresas expresada anteriormente se utiliza como denominador el conjunto de compañías existentes en el año anterior al de la tasa estimada. Este procedimiento es particularmente importante para analizar la renovación de la base de emprendimientos, dado que la tasa resultante mide la proporción del conjunto que representa el nuevo tejido. En general, ese indicador se expresa con relación a 100 empresas ya existentes. De acuerdo con Johnson (2004), a esta forma de medir la tasa de creación de empresas se la refiere como tasa de creación ecológica (ecological approach). En una medida alternativa - designada como tasa de creación relativa a la fuerza de trabajo o enfoque del mercado laboral (labor market approach) y propuesta por Armington y Acs (2002) - se utiliza la población (o fuerza de trabajo) como denominador. La tasa de creación de empresas relativa a la fuerza de trabajo es más relevante para examinar el grado de espíritu empresarial de una población local, o sea, determinar la proporción de habitantes involucrada en la creación de un nuevo negocio. Entonces, por ejemplo, la especificación alternativa para la tasa de creación de empresas en la región $r$ agregada para todos los sectores, denotada aquí como Fir* $^{*}$, puede definirse como:

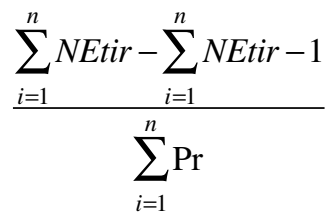

donde Pr es una medida de la población o de la fuerza de trabajo en la región $r$. Obviamente, para el cálculo de la tasa de creación de empresas de un determinado sector solo se utiliza la fuerza de trabajo ocupada en dicho sector. Este indicador se expresa en relación con 1.000 empleados en la región, de modo que un $\mathrm{Fir}^{*}=$ 10 significa que se formaron 10 nuevas empresas por cada 1.000 trabajadores en la región.

Por último, para la realización de la prueba empírica de la tasa de creación de empresas en el crecimiento económico se probó el siguiente modelo:

$$
\Delta P I B=c+P I B_{i, t}+T M_{i, t}+T X_{i, t}+\varepsilon_{i, t}
$$

donde $i$ representa el municipio y $\triangle P I B$ la variación del producto interno bruto (PIB) municipal. Debido a que la falta de datos del PIB municipal con respecto a todos los años de la serie de este trabajo impidió el uso de la metodología de datos de panel, se optó por probar el modelo anterior con datos transversales relativos a algunos años. El modelo expresa, por lo tanto, si la tasa de creación de empresas $T X_{i, t}$ de 2002 a 2005 tiene repercusiones en el crecimiento medio del PIB municipal entre 2005 y 2007. Las variables de control PIB $_{i, t}$ y PIBPC i,t $_{i}$ representan el PIB y el PIB per cápita en el año 2001 y $T M_{i, t}$ es el tamaño medio de las empresas en cada municipio de 2002 a 2005.

\section{IV}

\section{Resultados y discusión}

En el cuadro 1 se presentan las dos tasas de creación de empresas en cada una de las microrregiones de Rio Grande do Sul. Las dos medidas producen diferencias en el ordenamiento regional y están poco correlacionadas $(r=0,08)$. Este resultado no concuerda con aquellos relativos a los Estados Unidos de América (Armington y Acs, 2002) y el Reino Unido de Gran Bretaña e Irlanda del Norte (Johnson, 2004). De manera sorprendente, ambas medidas revelan altas tasas de creación de empresas en algunas microrregiones que no están entre las más desarrolladas del estado, entre ellas Soledade, Frederico Westphalen, Osorio y Cerro Largo. Por el contrario, otras microrregiones como Caxias do Sul,
Porto Alegre y Santa Cruz do Sul presentan bajas tasas de creación de empresas, no obstante su ubicación entre las de mayor nivel de desarrollo del estado. Obviamente, las dos medidas tienen distintas repercusiones políticas. Por ejemplo, si la meta de la microrregión Caxias do Sul en 2007 hubiese sido alcanzar la misma tasa de creación de nuevas empresas que el resto del estado, esto hubiera requerido la fundación de 225 nuevas empresas según el método ecológico y de 505 nuevas empresas conforme con el método alternativo, Fir*.

Gráficamente, puede apreciarse que el estado se concentra en Porto Alegre y Caxias do Sul. Sin embargo, 
CUADRO 1

Rio Grande do Sul: tasas de creación de empresas, especificaciones alternativas, 1996-2007

\begin{tabular}{|c|c|c|c|c|}
\hline Microrregión & $\begin{array}{c}\text { Fir } \\
\text { (porcentajes) }\end{array}$ & $\begin{array}{c}\text { Fir }^{*} \\
\text { (porcentajes) }\end{array}$ & $\begin{array}{l}\text { Conjunto medio de } \\
\text { empresas (unidades) }\end{array}$ & $\begin{array}{l}\text { PIB per cápita } 2005 \\
\text { (en miles de reales) }\end{array}$ \\
\hline Cachoeira do Sul & 13,4 & 13,4 & 2159,8 & 7,9 \\
\hline Camaquã & 14,3 & 12,8 & 1441,7 & 8,8 \\
\hline Campanha Central & 12,8 & 15,6 & 3134,2 & 6,7 \\
\hline Campanha Meridional & 12,8 & 13,9 & 2710,7 & 8,5 \\
\hline Campanha Ocidental & 13,5 & 13,4 & 5280,0 & 9,1 \\
\hline Carazinho & 14,2 & 13,0 & 2560,1 & 9,4 \\
\hline Caxias do Sul & 14,1 & 8,7 & 15003,0 & 19,5 \\
\hline Cerro Largo & 16,0 & 20,5 & 913,0 & 8,5 \\
\hline Cruz Alta & 14,0 & 16,2 & 2436,5 & 9,9 \\
\hline Erechim & 14,3 & 10,3 & 3222,6 & 11,3 \\
\hline Frederico Westphalen & 17,1 & 20,7 & 1992,8 & 7,4 \\
\hline Gramado-Canela & 16,7 & 8,7 & 5379,2 & 11,3 \\
\hline Guaporé & 15,9 & 12,0 & 2214,4 & 16,5 \\
\hline Ijuí & 14,6 & 12,8 & 2954,2 & 11,3 \\
\hline Jaguarão & 13,7 & 16,5 & 947,7 & 7,0 \\
\hline Lajeado-Estrela & 15,7 & 9,6 & 5234,5 & 14,4 \\
\hline Litoral Lagunar & 15,6 & 10,1 & 2956,5 & 12,0 \\
\hline Montenegro & 15,6 & 8,6 & 2951,2 & 14,3 \\
\hline Não-me-Toque & 13,5 & 14,4 & 882,7 & 13,4 \\
\hline Osório & 19,0 & 17,5 & 5112,7 & 8,1 \\
\hline Passo Fundo & 15,6 & 12,5 & 5584,4 & 13,4 \\
\hline Pelotas & 14,2 & 10,4 & 6083,0 & 7,5 \\
\hline Porto Alegre & 15,4 & 7,2 & 53237,9 & 16,1 \\
\hline Restinga Seca & 13,6 & 16,4 & 896,6 & 8,5 \\
\hline Sananduva & 14,8 & 18,8 & 757,2 & 8,4 \\
\hline Santa Cruz do Sul & 15,1 & 8,4 & 4143,1 & 16,7 \\
\hline Santa Maria & 15,1 & 13,4 & 5517,2 & 8,5 \\
\hline Santa Rosa & 14,8 & 14,0 & 2438,2 & 10,2 \\
\hline Santiago & 14,3 & 16,9 & 1018,0 & 8,0 \\
\hline Santo Ângelo & 14,4 & 15,7 & 2874,1 & 7,8 \\
\hline São Jerônimo & 16,3 & 9,7 & 1429,2 & 45,2 \\
\hline Serras de Sudeste & 14,9 & 13,6 & 1441,6 & 8,3 \\
\hline Soledade & 17,2 & 19,5 & 791,5 & 6,6 \\
\hline Três Passos & 16,2 & 15,1 & 1935,6 & 11,0 \\
\hline Vacaria & 15,4 & 9,1 & 2719,1 & 9,7 \\
\hline Media & 15,1 & 9,5 & 160995,7 & 13,3 \\
\hline
\end{tabular}

Fuente: elaboración propia sobre la base de la Relación Anual de Informaciones Sociales (RAIS).

esa concentración tiende a disminuir especialmente porque dichas microrregiones presentan menores tasas de creación de empresas que las demás. Por otra parte, se observa el rápido crecimiento de regiones otrora menos dinámicas, que comienzan a ganar espacio en el escenario estadual y a consolidar de ese modo un proceso de convergencia de la estructura empresarial en Rio Grande do Sul.

Un hecho importante que se desprende de los datos es la considerable variación en la tasa de creación de empresas entre los sectores. En el cuadro 2 se detallan las tasas Fir y Fir* relativas a los 16 sectores analizados. Una vez más los valores varían de acuerdo con las medidas utilizadas, pero se observa una mayor correlación de los dos índices a nivel sectorial que a nivel microrregional $(r=0,45)$. La menor tasa de creación de empresas según ambos índices se registró en el sector de administración pública, defensa y seguridad social, seguido por el sector de electricidad y gas. Por otra parte, las tasas más altas se encontraron en los sectores de comercio y reparación de vehículos, de organismos internacionales y otras instituciones extraterritoriales, y de alojamiento y alimentación.

Como ya se mencionó, las tasas de creación de empresas no son homogéneas a nivel microrregional ni sectorial, siendo potencialmente mayores o menores en las áreas con mayor o menor proporción de sectores con altas tasas de creación de empresas, respectivamente. La variación regional en la localización de los sectores con diferentes tasas de creación de empresas puede reflejar diferencias en las ventajas competitivas regionales, pero también, como señala Johnson (2005), puede ser resultado de las dependencias de trayectoria 
GRÁFICO 2

Rio Grande do Sul: tasas de creación de empresas y número de empresas (conjunto anual medio) por microrregión, 1996-2007

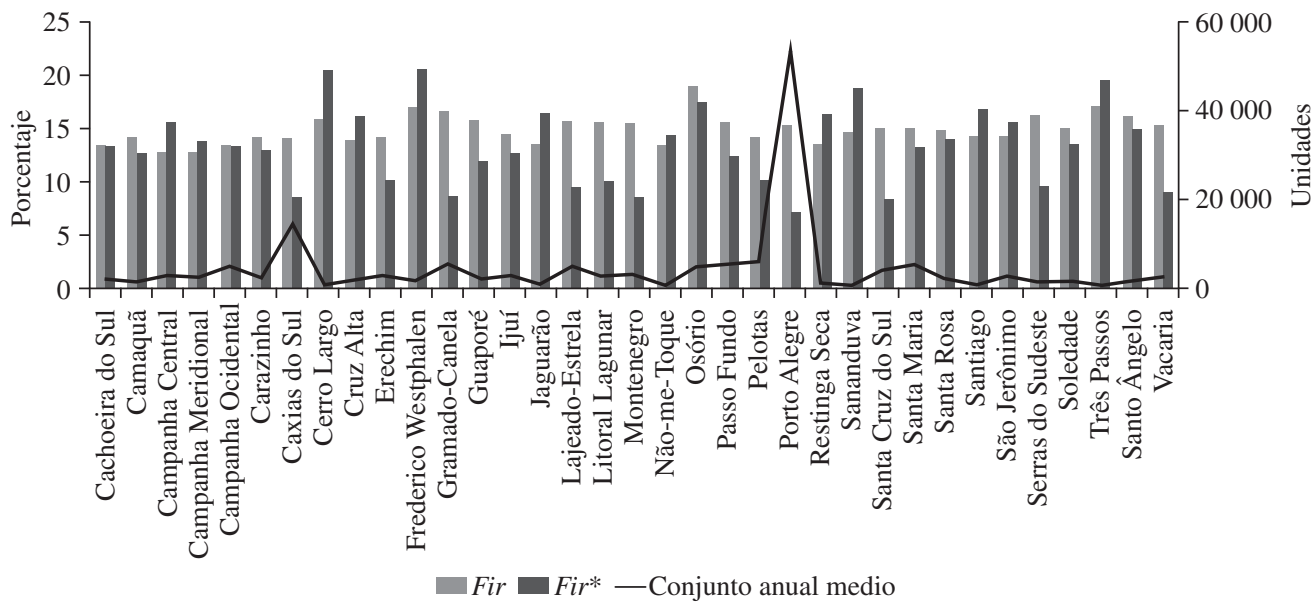

Fuente: Elaboración propia, sobre la base de la Relación Anual de Informaciones Sociales (RAIS).

CUADRO 2

Rio Grande do Sul: tasas de creación de empresas por sector, 1996-2007

(En porcentajes)

\begin{tabular}{|c|c|c|}
\hline Sectores & Fir & Fir* \\
\hline Agricultura, ganadería, producción forestal, pesca y acuicultura & 11,1 & 16,0 \\
\hline Industria de transformación & 13,3 & 4,0 \\
\hline Electricidad y gas & 8,0 & 2,3 \\
\hline Agua, alcantarillado, actividades de gestión de residuos y descontaminación & 12,0 & 5,4 \\
\hline Comercio; reparación de vehículos automotores y motocicletas & 47,0 & 25,3 \\
\hline Transporte, almacenamiento y correo & 14,2 & 18,2 \\
\hline Alojamiento y alimentación & 18,3 & 18,5 \\
\hline Información y comunicación & 13,9 & 8,6 \\
\hline Actividades financieras, de seguros y servicios relacionados & 10,2 & 5,2 \\
\hline Actividades profesionales, científicas y técnicas & 12,4 & 10,7 \\
\hline Actividades administrativas y servicios complementarios & 21,4 & 5,8 \\
\hline Administración pública, defensa y seguridad social & 4,3 & 0,1 \\
\hline Educación & 10,0 & 17,3 \\
\hline Salud humana y servicios sociales & 18,6 & 2,7 \\
\hline Artes, cultura, deporte y recreación & 9,9 & 9,4 \\
\hline Organismos internacionales y otras instituciones extraterritoriales & 18,0 & 42,6 \\
\hline Total & 15,1 & 9,5 \\
\hline
\end{tabular}

Fuente: elaboración propia sobre la base de la Relación Anual de Informaciones Sociales (RAIs).

Nota: Las categorías industrias extractivas, construcción civil, actividades inmobiliarias, otras actividades de servicios y servicios domésticos no están incluidas como categorías separadas, pues solo aparecen así en la RAIs a partir de 2006.

(path dependency), la disponibilidad de oportunidades, el modo en que se perciben las oportunidades y la oferta de emprendedores potenciales.

Al comparar las tasas de creación de empresas de regiones consideradas menos dinámicas con las tasas registradas en el estado como un todo, se observa que existe una gran variabilidad en los resultados (véase el cuadro 3). No obstante, se puede apreciar la relación entre dinamicidad económica y tasa de creación de empresas. Por ejemplo, en el caso de las microrregiones localizadas en la que convencionalmente se denomina "mitad sur", la tasa de creación de empresas 
CUADRO 3

Rio Grande do Sul: tasas de creación de empresas por microrregiones, por sector y total con respecto a la media estadual, 1996-2008

(En porcentajes)

\begin{tabular}{|c|c|c|c|c|c|c|c|c|c|}
\hline \multirow{2}{*}{$\begin{array}{c}\text { Parte del } \\
\text { estado }\end{array}$} & \multirow{2}{*}{ Microrregiones } & \multicolumn{4}{|c|}{ Fir } & \multicolumn{4}{|c|}{ Fir $^{*}$} \\
\hline & & Agricultura & Industria & Comercio & Total & Agricultura & Industria & Comercio & Total \\
\hline \multirow{24}{*}{ Mitad norte } & Camaquã & 1,03 & 0,92 & 0,99 & 0,95 & 1,04 & 1,55 & 1,27 & 1,34 \\
\hline & Carazinho & 0,62 & 1,00 & 1,04 & 0,94 & 0,73 & 1,57 & 1,34 & 1,37 \\
\hline & Caxias do Sul & 1,69 & 0,83 & 0,92 & 0,93 & 1,48 & 0,94 & 1,03 & 0,91 \\
\hline & Cerro Largo & 1,12 & 0,91 & 1,07 & 1,06 & 2,35 & 3,15 & 1,88 & 2,15 \\
\hline & Cruz Alta & 0,73 & 0,90 & 1,04 & 0,93 & 1,22 & 2,32 & 1,49 & 1,71 \\
\hline & Erechim & 1,06 & 0,95 & 0,94 & 0,95 & 1,72 & 1,11 & 1,09 & 1,08 \\
\hline & Frederico Westphalen & 1,16 & 1,22 & 1,10 & 1,13 & 2,28 & 4,04 & 1,87 & 2,17 \\
\hline & Gramado-Canela & 1,56 & 1,22 & 1,06 & 1,11 & 2,41 & 0,93 & 1,37 & 0,92 \\
\hline & Guaporé & 1,59 & 0,98 & 1,05 & 1,05 & 1,60 & 1,40 & 1,50 & 1,26 \\
\hline & Ijuí & 0,80 & 0,87 & 1,01 & 0,97 & 1,04 & 1,23 & 1,35 & 1,34 \\
\hline & Lajeado-Estrela & 1,23 & 1,01 & 1,03 & 1,04 & 0,92 & 0,69 & 1,46 & 1,00 \\
\hline & Montenegro & 1,45 & 1,00 & 1,01 & 1,03 & 1,49 & 0,70 & 1,29 & 0,91 \\
\hline & Não-me-Toque & 0,67 & 0,81 & 1,02 & 0,89 & 1,20 & 1,08 & 1,63 & 1,51 \\
\hline & Osorio & 1,12 & 1,32 & 1,24 & 1,26 & 1,25 & 2,07 & 1,73 & 1,84 \\
\hline & Passo Fundo & 0,93 & 0,99 & 1,04 & 1,03 & 1,38 & 1,13 & 1,34 & 1,31 \\
\hline & Porto Alegre & 1,18 & 1,08 & 0,98 & 1,02 & 1,19 & 0,92 & 0,72 & 0,75 \\
\hline & Sananduva & 0,93 & 0,95 & 1,01 & 0,98 & 2,16 & 2,88 & 1,73 & 1,98 \\
\hline & Santa Cruz do Sul & 1,00 & 0,93 & 0,99 & 1,00 & 0,86 & 0,54 & 1,19 & 0,89 \\
\hline & Santa Rosa & 0,94 & 0,99 & 0,98 & 0,98 & 1,54 & 1,73 & 1,38 & 1,47 \\
\hline & Santo Ângelo & 0,90 & 1,02 & 1,00 & 0,95 & 2,00 & 1,99 & 1,37 & 1,65 \\
\hline & Soledade & 0,99 & 1,31 & 1,16 & 1,14 & 1,49 & 4,26 & 1,74 & 2,05 \\
\hline & Tres Passos & 1,05 & 1,05 & 1,07 & 1,07 & 2,01 & 1,51 & 1,54 & 1,58 \\
\hline & Vacaria & 1,34 & 0,88 & 1,03 & 1,02 & 0,28 & 1,63 & 1,51 & 0,96 \\
\hline & Total norte & 1,08 & $\mathbf{1 , 0 2}$ & 1,00 & 1,02 & $\mathbf{0 , 9 3}$ & 0,99 & 0,96 & 0,94 \\
\hline \multirow{13}{*}{ Mitad sur } & Santiago & 0,85 & 0,95 & 1,09 & 0,95 & 1,45 & 1,61 & 1,54 & 1,77 \\
\hline & Santa Maria & 0,94 & 0,86 & 1,02 & 1,00 & 2,12 & 1,53 & 1,19 & 1,40 \\
\hline & Restinga Seca & 0,79 & 0,76 & 1,01 & 0,90 & 2,49 & 1,78 & 1,56 & 1,72 \\
\hline & Cachoeira do Sul & 0,85 & 0,78 & 0,98 & 0,89 & 1,41 & 1,50 & 1,26 & 1,40 \\
\hline & São Jerônimo & 1,25 & 1,28 & 1,05 & 1,08 & 0,69 & 0,81 & 1,15 & 1,02 \\
\hline & Campanha Central & 0,77 & 0,84 & 0,95 & 0,85 & 1,06 & 1,93 & 1,43 & 1,64 \\
\hline & Campanha Meridional & 0,91 & 0,89 & 0,92 & 0,85 & 1,27 & 1,52 & 1,17 & 1,46 \\
\hline & Campanha Ocidental & 0,93 & 0,90 & 0,96 & 0,89 & 1,01 & 1,32 & 1,21 & 1,41 \\
\hline & Serras de Sudeste & 1,18 & 0,99 & 1,02 & 0,98 & 1,11 & 0,85 & 1,43 & 1,43 \\
\hline & Pelotas & 0,97 & 0,80 & 0,95 & 0,94 & 1,06 & 0,88 & 1,07 & 1,09 \\
\hline & Jaguarão & 0,99 & 1,05 & 0,97 & 0,90 & 0,98 & 4,31 & 1,51 & 1,73 \\
\hline & Litoral Lagunar & 1,02 & 0,99 & 1,03 & 1,03 & 0,52 & 0,72 & 1,07 & 1,06 \\
\hline & Total sur & 0,93 & $\mathbf{0 , 8 8}$ & 0,99 & 0,94 & 1,10 & 1,13 & $\mathbf{1 , 2 0}$ & 1,32 \\
\hline Total general & & 1,00 & 1,00 & 1,00 & 1,00 & 1,00 & 1,00 & 1,00 & 1,00 \\
\hline
\end{tabular}

Fuente: elaboración propia sobre la base de la Relación Anual de Informaciones Sociales (RAIS).

relativa al conjunto de ellas (Fir) es de apenas el 94\% de la registrada con respecto al estado considerado globalmente. Por otra parte, la tasa de creación de empresas relativa a la fuerza de trabajo $\left(\right.$ Fir $\left.^{*}\right)$ es un $32 \%$ mayor que la media estadual, lo que indica que el espíritu empresarial de los trabajadores de la mitad sur es mayor que el de sus pares de la mitad norte. En consecuencia, si bien en la mitad norte hay una mayor renovación de la base empresarial, se registra al mismo tiempo un menor índice de creación de empresas por trabajador, mientras que en la mitad sur ocurre exactamente lo contrario.
En el gráfico 3 se muestran las dos tasas de creación de empresas respecto de las mitades norte y sur de Rio Grande do Sul en el período 1996-2007. La trayectoria de las tasas Fir y Fir* es similar en las dos unidades geográficas, con una pequeña disminución a lo largo del período. Aunque en promedio los residentes de la mitad sur son menos emprendedores que los trabajadores del estado considerado globalmente, en el gráfico 3 puede observarse que este desfase disminuye con el tiempo, tanto que en el año 2007 las tasas Fir son prácticamente iguales en las dos regiones. Con respecto a la tasa Fir* se observa cierta 


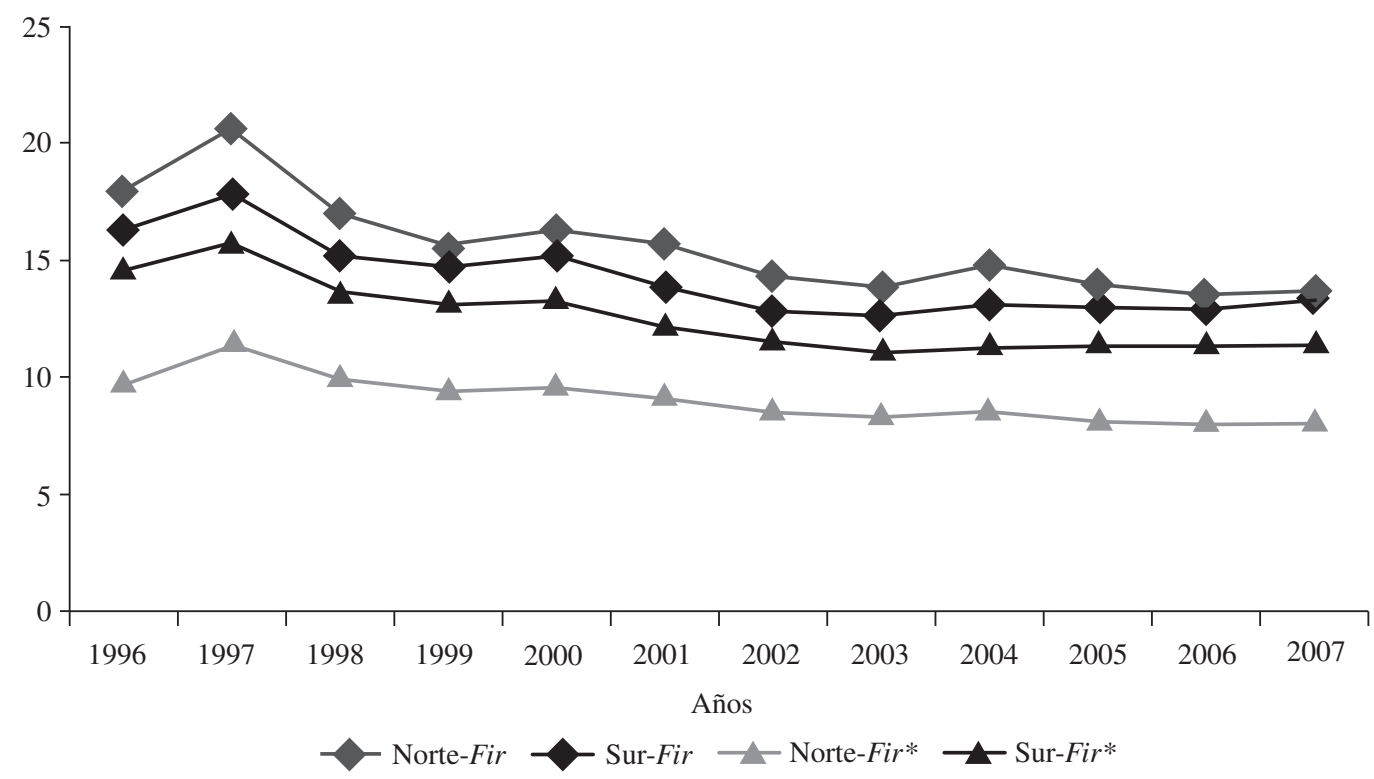

Fuente: elaboración propia sobre la base de la Relación Anual de Informaciones Sociales (RAIS).

estabilidad en la mitad sur, mientras que en la mitad norte se aprecia una pequeña pero constante reducción. De estos resultados se desprende que el menor nivel de desarrollo económico de la mitad sur no se relaciona con el impulso emprendedor reciente de los trabajadores locales, pero todavía refleja un desfase histórico de la base empresarial. Esto significa que en la mitad sur se debe acelerar el ritmo de la creación de empresas con respecto al conjunto de empresas ya existentes para alcanzar el nivel de desarrollo del resto del estado. Sin embargo, esta es solo una hipótesis que merece un análisis más profundo, pues el efecto también puede ser el contrario. En otras palabras, un alto nivel de renovación empresarial en una determinada región puede indicar que esta región todavía no tiene industrias y sectores consolidados, así como la falta de definición de su vocación competitiva que se traduce en una aminoración del crecimiento económico.

El efecto de la creación de empresas en el crecimiento de la actividad económica ha sido una de las principales justificaciones de los proyectos concernientes al espíritu empresarial. En el cuadro 4 se ilustra que el supuesto efecto tiene lugar de hecho. Pero si bien existe, no es un efecto simple y constante. Como puede observarse en las dos últimas columnas del cuadro 4 , inicialmente la tasa de creación de empresas se relaciona en forma negativa con el crecimiento económico municipal (medido a través de la variación del PIB entre 2005 y 2007). Sin embargo, dicha relación no es significativa. Esto quiere decir que las altas tasas de creación de empresas no son eficaces para impulsar el desarrollo en el corto plazo y solo después de un determinado período las empresas entrantes podrán influir en el desempeño económico regional con la introducción satisfactoria de nuevas soluciones, métodos, procesos y productos. La relación negativa también puede denotar una reducción de la productividad de la economía regional en el corto plazo, debido a la mala asignación de recursos en empresas nuevas todavía ineficientes y con grandes probabilidades de cerrar.

Por otra parte, los resultados también resaltan que con el pasar de los años el efecto se vuelve positivo y significativo. De ese modo, un aumento en la tasa de creación de empresas en un año cualquiera $-n$, por ejemplo - pasa a relacionarse en forma positiva con la expansión del PIB en el año $n+1$. El efecto alcanza el punto máximo un año después de efectuada la expansión de la tasa de creación de empresas - Fir*_- y se vuelve decreciente en los años subsiguientes. El efecto máximo está más retardado con respecto a la tasa Fir, pues se mantiene positivo y significativo hasta el cuarto y quinto años después de la expansión. 
CUADRO 4

Rio Grande do Sul: efecto de la creación de empresas en la variación media del producto interno bruto municipal, 2005-2007

(Coeficientes de regresión obtenidos de la ecuación 4)

\begin{tabular}{|c|c|c|c|c|c|c|c|c|}
\hline \multirow{2}{*}{ Variables } & \multicolumn{2}{|c|}{2002} & \multicolumn{2}{|c|}{2003} & \multicolumn{2}{|c|}{2004} & \multicolumn{2}{|c|}{2005} \\
\hline & Fir & Fir ${ }^{*}$ & Fir & $\mathrm{Fir}^{*}$ & Fir & Fir $^{*}$ & Fir & $\mathrm{Fir}^{*}$ \\
\hline PIB municipal 2001 & $\begin{array}{l}-, 419 * * * \\
(-9,00)\end{array}$ & $\begin{array}{l}-, 416 * * * \\
(-9,01)\end{array}$ & $\begin{array}{l}-, 423 * * * \\
(-9,06)\end{array}$ & $\begin{array}{l}-, 427 * * * \\
(-9,31)\end{array}$ & $\begin{array}{l}-, 430 * * * \\
(-9,39)\end{array}$ & $\begin{array}{l}-, 415^{* * * *} \\
(-9,09)\end{array}$ & $\begin{array}{c}-, 422^{* * * *} \\
(-9,25)\end{array}$ & $\begin{array}{l}-, 422 * * * \\
(-9,26)\end{array}$ \\
\hline PIB per cápita 2001 & $\begin{array}{l}, 240 * * * \\
(5,21)\end{array}$ & $\begin{array}{c}, 236 * * * \\
(5,14)\end{array}$ & $\begin{array}{l}, 235 * * * \\
(5,07)\end{array}$ & $\begin{array}{l}, 240 * * * \\
(5,20)\end{array}$ & $\begin{array}{l}, 242^{* * * *} \\
(5,24)\end{array}$ & $\begin{array}{l}, 242 * * * \\
(5,29)\end{array}$ & $\begin{array}{l}, 250 * * * \\
(5,41)\end{array}$ & $\begin{array}{l}, 250 * * * \\
(5,43)\end{array}$ \\
\hline $\begin{array}{l}\text { Tamaño medio } \\
\text { Tasa de creación }\end{array}$ & $\begin{array}{c}-, 177 * * * \\
(-4,23) \\
, 090 * * \\
(2,13)\end{array}$ & $\begin{array}{c}-, 110 * * \\
(-2,43) \\
, 127 * * \\
(2,76)\end{array}$ & $\begin{array}{c}-, 147 * * * \\
(-3,52) \\
, 078 * \\
(1,85)\end{array}$ & $\begin{array}{c}-, 084 * \\
(,-1,84) \\
, 131 * * \\
(2,85)\end{array}$ & $\begin{array}{c}-, 182^{* * * *} \\
(-4,33) \\
, 046 \\
(1,11)\end{array}$ & $\begin{array}{c}-, 097 * * \\
(-2,03) \\
, 155 * * * \\
(3,24)\end{array}$ & $\begin{array}{c}-, 220 * * * \\
(-5,12) \\
-, 006 \\
(-, 13)\end{array}$ & $\begin{array}{c}-, 228 * * * \\
(-4,70) \\
-, 013 \\
(-, 27)\end{array}$ \\
\hline $\begin{array}{l}\text { Número de } \\
\text { observaciones }\end{array}$ & 494 & 494 & 494 & 494 & 494 & 494 & 494 & 494 \\
\hline $\begin{array}{l}\mathrm{R}^{2} \text { ajustado } \\
\text { Prueba F }\end{array}$ & $\begin{array}{r}, 18 \\
28,62\end{array}$ & $\begin{array}{r}, 18 \\
29,57\end{array}$ & $\begin{array}{r}, 17 \\
26,99\end{array}$ & $\begin{array}{r}, 18 \\
28,42\end{array}$ & $\begin{array}{r}, 18 \\
28,35\end{array}$ & $\begin{array}{r}, 19 \\
31,19\end{array}$ & $\begin{array}{r}, 19 \\
31,06\end{array}$ & $\begin{array}{r}, 19 \\
31,07\end{array}$ \\
\hline
\end{tabular}

Fuente: elaboración propia sobre la base del Instituto Brasileño de Geografía y Estadística (IBGE).

Nota 1 - Coeficientes beta, valores de $t$ entre paréntesis; $*<, 10, * *<, 05 * * *<, 001$

El efecto de la tasa de creación de empresas en el desempeño económico puede, entonces, no ser constante en el tiempo. Audretsch y Fritsch (2002) encontraron resultados similares a los de este estudio, que también recuerda las etapas de reacción del mercado propuestas por Carree y Thurik (2006), quienes reconocen que en los años directamente posteriores a la entrada de nuevas empresas se observa una disminución de ganancias. Esto se debe a la elevada tasa de desaparición de las nuevas empresas y a los efectos de sustitución de las empresas ya existentes. Después del primer año de funcionamiento la economía se vuelve más competitiva, tanto debido a las mejoras de los procesos de producción como de los productos, y esto se traduce en un incremento del desempeño económico. En este período, las empresas sobrevivientes comienzan a contribuir realmente al crecimiento económico y social.

\section{Consideraciones finales}

En este trabajo se analizó la dinámica de la creación de nuevas empresas en Rio Grande do Sul mediante la tasa de creación relativa al conjunto de empresas ya existentes y la tasa relativa a la fuerza de trabajo de cada sector económico. La descomposición de la tasa de creación de nuevas empresas de la manera presentada en este artículo puede, por ejemplo, ayudar a determinar el origen de las diferencias entre una región específica y el resto del estado. De este modo, en los análisis referidos a la mitad sur del estado se pudo constatar que si bien existen diferencias en cuanto al nivel de desarrollo, estas se vinculan a un desfase en la capacidad de renovar la base empresarial con respecto a todo el estado. Por otra parte, la tasa de creación de empresas cada 1.000 personas ocupadas en la mitad sur es mayor que la media de Rio Grande do Sul, lo que potencialmente conducirá a la convergencia entre las regiones. Obviamente, una consecuencia directa de este resultado es que el análisis de la dinámica del ingreso en los municipios, sobre todo con respecto al proceso de convergencia, será más sólido si se tiene en cuenta el fenómeno de creación de empresas entre las diferentes regiones del estado.

Si las tasas de creación de nuevas empresas no son homogéneas regionalmente, tampoco lo son a nivel sectorial. 
Esto lleva a pensar que las diferencias regionales pueden no depender únicamente de diferencias entre las regiones en la creación de empresas dentro de un mismo sector, sino de diferencias en la estructura sectorial entre las regiones. En otras palabras, la estructura sectorial de una región puede ser más o menos favorable para la creación de nuevas empresas. Sin embargo, como en este trabajo no se descompuso el efecto de la estructura sectorial en la tasa de creación de empresas, no es posible afirmar que las diferencias entre una región y otra dependen en mayor medida de la tasa de creación de empresas en un mismo sector o de las diferencias en la estructura sectorial entre las regiones. Ciertamente, se trata de un aspecto que debe investigarse en futuros estudios.

Si bien todavía queda mucho por examinar con respecto a la importancia del espíritu empresarial y particularmente de la creación de nuevas empresas en el progreso regional, este trabajo constituye un marco inicial dada la escasez de estudios sobre el tema en el Brasil. Como es obvio, la profundización en esta área puede ser de gran ayuda para la comprensión de las diferencias regionales y la formulación de políticas.
En ese sentido, el hecho de que la tasa de creación de nuevas empresas esté relacionada con la creación de riqueza (como se demuestra en el cuadro 4), revela que las políticas destinadas a mejorar el impulso emprendedor y la calidad del espíritu empresarial pueden tener efectos significativos en el desarrollo regional. Pese a que esta relación necesita controlarse mediante otras variables en futuros estudios para evitar correlaciones espurias, este trabajo es - según tienen entendido sus autores-el primero en demostrar empíricamente la relación entre la tasa de creación de nuevas empresas y las variables de desempeño como el PIB en el Brasil.

También se deben reconocer limitaciones con relación a los datos y al nivel de agregación de los sectores. No obstante las carencias, este estudio se beneficiará de futuras investigaciones para la mejor comprensión de las diferencias regionales en sectores específicos. Además, también se necesita analizar las variaciones regionales en lo que se refiere a la calidad de la creación de nuevas empresas, principalmente en términos de la supervivencia y el crecimiento de los emprendimientos.

\section{Bibliografía}

Acs, Z. y D. Audretsch (2003), The International Handbook of Entrepreneurship, Dordrecht, Kluwer Academic Publishers.

Aghion, P. y P. Howitt (1998), Endogenous Growth Theory, Cambridge, The MIT Press.

Armington, C. y Z.J. Acs (2002), “The determinants of regional variation in new firm formation", Regional Studies, vol. 36, $\mathrm{N}^{\circ} 1$, Londres, Taylor and Francis.

Ashcroft, B., M. Plotnikova y D. Ritchie (2007), "New firm formation in British counties: comparing the 1990s with the 1980s", CPPR Working Paper, $\mathrm{N}^{\circ}$ 6, Glasgow, Centre for Public Policy for Regions (CPPR).

Audretsch, D.B. (2007), "Entrepreneurship capital and economic growth", Oxford Review of Economic Policy, vol. 23, $\mathrm{N}^{\circ} 1$, Oxford, Oxford University Press.

(1999), "Linking entrepreneurship to economic growth", Advances in the Study of Entrepreneurship and Economic Growth, G.D. Libecap (ed.), vol. 11, Stanford, JAI Press.

Audretsch, D.B. y M. Fritsch (2002), "Growth regimes over time and space”, Regional Studies, vol. 36, № 2, Londres, Taylor and Francis.

Audretsch, D.B. y M. Keilbach (2007), "Entrepreneurship capital and economic growth", Oxford Review of Economic Policy, vol. 23, $\mathrm{N}^{\mathrm{o}}$ 1, Oxford, Oxford University Press.

Barros, A.A. y C.M.M.A. Pereira (2008), "Empreendedorismo e crescimento econômico: uma análise empírica", Revista de administração contemporânea, vol. 12, $\mathrm{N}^{\circ}$ 4, Curitiba, Associação Nacional de Pós-Graduação e Pesquisa em Administração (ANPAD).

Campos, N.F. y M. Iootty (2005), "Firm entry and exit in Brazil: cross-sectoral evidence from manufacturing industry", Anais do XXXIII Encontro Nacional de Economia, No 095, Asociación Nacional de Centros de Posgrado en Economía (ANPEC).

Canever, M.D. y otros (2010), "Entrepreneurship in the Rio Grande do Sul, Brazil: the determinants and consequences for the municipal development", Revista de economia e sociologia rural, vol. 48, Brasilia, Sociedad Brasileña de Economía y Sociología Rural. Carree, M. y R. Thurik (2006), "The lag structure of the impact of business ownership on economic performance in OECD countries", Research Paper, N $^{\circ}$ ERS-2006-064-ORG, Erasmus Research Institute of Management (ERIM).

Casarotto Filho, N. y L.H. Pires (2001), Redes de pequenas e médias empresas e desenvolvimento local, São Paulo, Atlas.

Cassiolato, J.E., H.M.M. Lastres y M. Szapiro (2000), “Arranjos e sistemas produtivos locais e proposições de políticas de desenvolvimento industrial e tecnológico", Nota técnica, $\mathrm{N}^{\circ} 27$, Río de Janeiro, Instituto de Economía de la Universidad Federal de Río de Janeiro.

Cassiolato, J.E. y M. Szapiro (2000), "Novos objetivos e instrumentos de política de desenvolvimento industrial e inovativo em países seleccionados", Nota técnica, N 13, Río de Janeiro, Universidad Federal de Río de Janeiro.

Castilhos, C.C. (2002), Programa de apoio aos sistemas locais de produção: a construção de uma política pública no RS, Porto Alegre, Fundación de Economía y Estadítica.

Cohen, W. y D. Levintahl (1989), "Innovation and learning: the two faces of R\&D”, Economic Journal, vol. 99, No 397, Royal Economic Society.

Feldmann, M. y D. Audretsch (1999), "Innovation in cities: science based diversity, socialization and localized competition", European Economic Review, vol. 43, № 2, Amsterdam, Elsevier.

Garther, W. y N. Carter (2003), "Entrepreneurship behavior: firm organizing processes", The International Handbook of Entrepreneurship, Z. Acs y D. Audretsch (eds.), Dordrecht, Kluwer Academic Publishers.

Glaeser, K. y otros (1992), "Growth of cities", Journal of Political Economy, vol. 100, № 6, Chicago, University of Chicago Press. 
Grossman, G. y E. Helpman (1991), Innovation and Growth in the Global Economy, Cambridge, The мIт Press.

Haddad, P.R.A. (1999), A competitividade do agronegócio e o desenvolvimento regional no Brasil; estudo de clusters, Brasilia, Consejo Nacional de Desarrollo Científico y Tecnológico (CNPq).

Hart, P.E. y N. Oulton (2001), "Galtonian regression, company age and job generation 1986-95", Scottish Journal of Political Economy, vol. 48, No 1 , Scottish Economic Society.

Herbert, R. y A.N. Link (1989), "In search of the meaning of entrepreneurship", Small Business Economics, vol. 1, N $\mathrm{N}^{\mathrm{o}} 1$, Springer.

Hofstede, G. (1980), Culture's Consequences: International Differences in Work Related Values, Beverly Hills, Sage Publications.

Hofstede, G. y otros (2002), "Culture's role in entrepreneurship", Innovation, Entrepreneurship and Culture: The Interaction between Technology, Progress and Economic Growth, J. Ulijn y T. Brown (eds.), Brookfield, Reino Unido, Edward Elgar.

Jacobs, J. (1969), The Economy of Cities, Nueva York, Random House.

Johnson, P. (2005), "Targeting firm births and economic regeneration in a lagging region", Small Business Economics, vol. 24, N ${ }^{\circ} 5$, Springer.

(2004), "Differences in regional firm formation rates: a decomposition analysis", Entrepreneurship Theory and Practice, vol. $28, \mathrm{~N}^{\mathrm{o}} 5$, Wiley.

Lastres, H.M.M., J.E. Cassiolato y M.L. Maciel (2003), "Systems of innovation for development in the knowledge era: an introduction", Systems of Innovation and Development: Evidence from Brazil, J.E. Cassiolato, H.M.M. Lastres y M.L. Maciel (eds.). Cheltenham, Edward Elgar.

Lucas, R. (1988), "On the mechanics of economic development", Journal of Monetary Economics, vol. 22, No 1, Amsterdam, Elsevier.

Mizumoto, F.M. y otros (2008), "O impacto de capital humano, capital social e práticas gerenciais na sobrevivência de empresas nascentes: um estudo com dados de pequenas empresas no Estado de São Paulo", IBMEC Working Paper, Nº 67, Instituto de Ensino e Pesquisa.

Najberg, S., F.P. Puga y P.A.D.S. Oliveira (2000), "Sobrevivências das firmas no Brasil: dez. 1995/dez. 1997”, Revista do BNDES, vol.7, $\mathrm{N}^{\circ}$ 13, Río de Janeiro, Banco Nacional de Desarrollo Econômico y Social (BNDES).

OCDE (Organización de Cooperación y Desarrollo Económicos) (1998), Fostering Entrepreneurship, París.

Paludo, O.F. (2008), "Empreendedor integrador - mobilizador de parcerias cooperativas inter empresariais que proporcionam um desenvolvimento regional sustentável - D.R.S.", tesis de doctorado, Florianópolis, Universidad Federal de Santa Catarina.
Porsse, A.A., J.A. Rosa y R.O. Porto (2008), Desenvolvimento e disparidades regionais no Rio Grande do Sul: sugestões de linhas de programas para dinamização de regiões de menor desenvolvimento relativo, Porto Alegre, Fundación de Economía y Estadística (FEE).

Porter, M. (1991), La ventaja comparativa de las naciones, Buenos Aires, Javier Vergara Editor.

Reynolds, P.D., D.J. Storey y P. Westhead (1994), "Cross-national comparisons of the variation in new firm formation rates", Regional Studies, vol. 28, No 4, Taylor and Francis.

Reynolds, P., W. Bygrave y E. Autio (2004), GEM 2003 Global Report, Babson College, Babson Park, MA.

Romer, P. (1990), "Endogenous technological change", Journal of Political Economy, vol. 98, No 5, Chicago, University of Chicago Press.

(1986), "Increasing returns and long-run growth", Journal of Political Economy, vol. 94, № 5, Chicago, Chicago University Press.

Schmitz, J.A. (1989), "Imitation, entrepreneurship, and long-run growth", Journal of Political Economy, vol. 97, № 3, Chicago, Chicago University Press.

Schumpeter, J.A. (1982), Teoria do desenvolvimento econômico, São Paulo, Editora Abril.

Sebrae-SP (Servicio Brasileño de Apoyo a las Micro y Pequeñas Empresas de São Paulo) (2005), Sobrevivência e mortalidade das empresas paulistas de 1 a 5 anos, São Paulo.

Solow, R.A. (1956), "Contribution to the theory of economic growth", Quartely Journal of Economics, vol. 70, № 1, Oxford University Press.

Souza, A.L. y otros (2007), "Base de dados: apresentação e opções metodológicas”, Criação, destruição e realocação de empregos no Brasil, C.H. Corseuil y L.M.S. Servo (orgs.), Brasilia, Instituto de Investigación Económica Aplicada (IPEA).

Thorton, P. y K. Flynne (2003), "Entrepreneurship, networks and geographies", The International Handbook of Entrepreneurship, Zoltan Acs y David Audretsch (eds.), Dordrecht, Kluwer Academic Publishers.

Thurik, A.R. (1999), "Entrepreneurship, industrial transformation and growth", Advances in the Study of Entrepreneurship and Economic Growth, G.D. Libecap (ed.), vol. 11, Stanford, JAI Press.

Van Stel, A., M. Carree y R. Thurik (2005), "The effect of entrepreneurial activity on national economic growth", Small Business Economics, vol. 24, No 3 , Springer.

Varian, H. (1992), Microeconomic Analysis, Nueva York, W.W. Norton.

Wennekers, S. y R. Thurik (1999), "Linking entrepreneurship and economic growth", Small Business Economic, vol. 13, No 1 , Springer. 